\title{
Association Study of Polymorphisms in Neuronal Nicotinic Acetylcholine Receptor Subunit Genes With Schizophrenia in the Han Chinese Population
}

\author{
Yuan-yuan $\mathrm{Li}^{1 *}$, Rui-jie Geng ${ }^{1 *}$, Shun-ying $\mathrm{Yu}^{2}$, Guan-jun $\mathrm{Li}^{2}$, Zhou-ye Wang ${ }^{3}$, and Hua-fang $\mathrm{Li}^{2} \bowtie$ \\ 1'Department of Psychological Medicine, Zhongshan Hospital, Fudan University, Shanghai, China \\ ${ }^{2}$ Department of Psychiatry, Shanghai Mental Health Center, Shanghai Jiao Tong University School of Medicine, Shanghai, China \\ ${ }^{3}$ Department of Medical Psychology, Xin Hua Hospital Affiliated to Shanghai Jiao Tong University School of Medicine, Shanghai, China
}

Objective To investigate the relation between nicotinic acetylcholine receptor subunit (nAChR) genes and schizophrenia, and the relation between tag single nucleotide polymorphism (rs1317286, rs1044396, rs6494212, rs16969968, and rs684513) and schizophrenia in Han Chinese people.

Methods The protein-protein interaction (PPI) network among nAChR protein and 350 proteins encoded by schizophrenia-related susceptibility genes was constructed through the String database to explore whether nAChR genes were associated with schizophrenia in these known databases. Then, five single nucleotide polymorphisms (SNPs) of CHRNA3 (rs1317286), CHRNA4 (rs1044396), CHRNA7 (rs6494212), and CHRNA5 (rs16969968, rs684513) were analyzed in a sample of 1,035 schizophrenic patients and 816 healthy controls. The interaction between the markers was analyzed using multifactor dimensionality reduction (MDR) software. Power analysis was performed using the Quanto program.

Results There are no significant differences in genotype or allele distribution were identified between the patients and controls ( $\mathrm{p}>0.05$ ). The haplotypes constructed by four markers rs1317286, rs6494212, rs16969968, and rs684513 were not associated with schizophrenia either. However, a significant association between models made of rs1317286, rs1044396, rs6494212, and rs684513 and schizophrenia was revealed in interaction analysis $(\mathrm{p}<0.05)$.

Conclusion The $\mathrm{nAChR}$ protein may have effects on the development of schizophrenia through the interaction with proteins encoded by schizophrenia-related susceptibility genes, but no relation was found between selected polymorphisms and schizophrenia in the collected Han Chinese people. However, interaction analysis suggested four-SNP model has an important effect on schizophrenia.

Psychiatry Investig 2021;18(10):943-948

Keywords Schizophrenia; Neuronal nicotinic acetylcholine receptor; Association study; Protein-protein interaction network.

\section{INTRODUCTION}

Schizophrenia is a complex neuropsychiatric disorder, af-

Received: May 5, 2021 Revised: July 6, 2021

Accepted: August 6, 2021

$\triangle$ Correspondence: Hua-fang $\mathrm{Li}, \mathrm{MD}, \mathrm{PhD}$

Department of Psychiatry, Shanghai Mental Health Center, Shanghai Jiao Tong University School of Medicine, 600 Wan Ping Nan Road, Shanghai 200030, China Tel: +86-021 34773107, Fax: +86-021 34773107, E-mail: lhlh_5@163.com

$\bowtie$ Correspondence: Zhou-ye Wang, MD, PhD

Department of Medical Psychology, Xin Hua Hospital Affiliated to Shanghai Jiao Tong University, School of Medicine, 1665 Kong Jiang Road, Shanghai 200092, China

Tel: +86-021 25076685, Fax: +021 25076685, E-mail: wzy_1021@126.com

*These authors contributed equally to this work.

(c) This is an Open Access article distributed under the terms of the Creative Commons Attribution Non-Commercial License (https://creativecommons.org/licenses/bync/4.0) which permits unrestricted non-commercial use, distribution, and reproduction in any medium, provided the original work is properly cited. fecting about $1 \%$ of the general population. ${ }^{1}$ This disease is characterized by a wide spectrum of symptoms, which include thought disorder, perceptual aberrations, cognitive difficulties, apathy and social withdrawal. Evidence from family, twin and adoption studies support high heritability in the development of schizophrenia, although the exact causes are unclear, genetic and environmental factors contribute to the etiology of schizophrenia. ${ }^{2}$

Schizophrenia has a higher frequency of smoking (over $80 \%$ ) than the normal population $(23 \%-30 \%))^{3,4}$ In addition, case reports showed that psychotic symptoms would relapse when patients stopped smoking. ${ }^{5}$ Nicotine has been reported to normalize two psychophysiological dysfunctions in schizophrenia: an auditory sensory gating deficit and abnormal smooth pursuit eye movement. ${ }^{6,7}$ Brain PET Imaging study 
found that the $\alpha 7$-nAChR was lower in the cingulate cortex, frontal cortex, and hippocampus of Schizophrenia patients. ${ }^{8}$ The diminished $\mathrm{nAChR}$ is a genetically transmitted trait related to the risk for schizophrenia, rather than the effect of smoking or antipsychotic treatment. ${ }^{910}$ As well known, typical antipsychotics are thought to exert their effects primarily by antagonizing dopamine $\mathrm{D} 2$ receptors leading to the dopamine hypothesis of schizophrenia. ${ }^{11}$ Pre-synaptic Nicotinic receptors can mediate the release of dopamine and this points to neuronal nicotinic receptors as important candidate genes in this disorder. ${ }^{12,13}$

Nicotine exerts its effect through neuronal nicotinic acetylcholine receptors expressed in the brain and periphery. Some of the nicotinic receptor subunit genes, such as CHRNA7 and CHRNA4 have been investigated the association with schizophrenia or other psychiatric disorders in genetic studies. ${ }^{14}$ The $\alpha 7$ neuronal nicotinic receptor subunit gene (CHRNA7), was first genetically linked to the P50 auditory sensory processing deficit in schizophrenia, and then to schizophrenia as a disease in multiple independent studies. ${ }^{15-17}$ Several studies have found decreased expression of the CHRNA7 gene in the postmortem hippocampus, reticular thalamic nucleus and frontal cortex of schizophrenic subjects. The $\alpha 7$ receptor agonists are ranked as the most promising targets for developing drugs to treat cognitive impairments in the disorder..$^{18}$ Recently, some studies indicated that variations in the gene CHRNA4 may modulate attention and spatial scaling performance in humans. ${ }^{19,20}$ Besides, CHRNA5 was found to be associated with nicotine dependence and CHRNA3 was found to be associated with prepulse inhibition (PPI). ${ }^{21,22}$

Therefore, to further evaluate the role of the genes above in susceptibility to schizophrenia, we conducted a case-control study in the Han Chinese population. We selected one SNP for CHRNA3, CHRNA4, CHRNA7, and two SNPs for CHRNA5 according to the previous studies and the minor allele frequency of the SNPs was higher than $0.05 .{ }^{19,23,24}$ Meanwhile, we explored the interactive effect among the SNPs we studied using the multifactor dimensionality reduction (MDR) method, which has great advantages versus the conventional statistical approaches for detecting gene-gene interactions of the genes selected. Complex interactions among genes and environmental factors are known to play a role in common human disease etiology. However, the identification and characterization of gene-gene interactions for common complex human diseases remain a challenge for human geneticists. Traditional statistical methods are not well suited for detecting such interactions, especially when the data are highly dimensional (having many attributes or independent variables) or when interactions occur between more than two polymorphisms. To address these issues, a variety of bioinformatics methods for identifying gene-gene interactions have been developed, and one such method is $\mathrm{MDR},{ }^{25}$ a data mining tool for detecting and characterizing nonadditive interactions between discrete variables that affect binary results.

\section{METHODS}

\section{Subject}

A total of 1,035 schizophrenic patients (526 males and 509 females) at Shanghai Mental Health Center, Shanghai Jiao

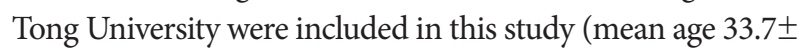
9.2 years). Diagnoses were made based on DSM-IV. Patients with other mental disorders and severe physical disorders were excluded. The control group consisted of 816 subjects (420 males and 396 females, mean age $33.3 \pm 10.6$ years). The control subjects were recruited from the local community in Shanghai. They self-reported to be free from mental disorders, family history of mental illness and severe physical disorders. All patients and controls were ethnically Chinese Han. The study protocol and process were assessed and approved by the ethics committee at Shanghai Mental Health Center (IRB No. 2019-16R). We obtained informed consent from each participant.

\section{Construction of protein-protein interaction network}

The Multiple proteins module of the CHRNA3, CHRNA4, CHRNA5, and CHRNA7 genes and 350 schizophrenia susceptibility genes identified in the Genome-wide Association Study (GWAS) of SZDB2.0 database (http://www.szdb.org) were uploaded to the String database (http://www.string-db. org/). ${ }^{26,27}$ A protein-protein interaction (PPI) network among the proteins encoded by these genes was constructed to determine the potential role of the four genes in the pathogenesis of schizophrenia. The String database is mainly used to obtain the PPI between gene-coding proteins by using previous data from different sources and can integrate the scores of different aspects of PPI to form a comprehensive score to represent the size of their interaction. In this study, the data of high confidence score of 0.7 was selected to construct the PPI network.

\section{SNP selection and genotyping}

A total of 5 SNPs within CHRNA3 (rs1317286), CHRNA4 (rs1044396), CHRNA7 (rs6494212), and CHRNA5 (rs16969968, rs684513) were selected based on the following criteria: 1) SNPs are capable of tagging more SNPs based on the linkage disequilibrium (LD) pattern of the respective genes according to the data from the HapMap CHB dataset (http://hapmap.ncbi. nlm.nih.gov/) and 1,000 Genomes (http://www.broadinstitute. org/mpg/snap/) and 2) all eligible SNPs should have a minor allele frequency (MAF) $>5 \%$ according to the HapMap CHB 
and dbSNP datasets (http://www.ncbi.nlm.nih.gov/SNP/).

Genomic DNA was isolated using a Tiangen DNA isolation kit (Tiangen Biotech, Beijing, China). Genotyping of the SNPs was carried out using the TaqMan SNP Genotyping Assay (Applied Biosystems, Foster City, CA, USA) on ABI PRISM7900 sequence detection system instrument (Applied Biosystems) and SDS 2.0 software (Applied Biosystems). For quality control, all genotypes were determined without knowledge of case or control status in the genotyping process. $5 \%$ of the samples were repeated for genotyping and the corresponding results were $100 \%$ concordant.

\section{Statistical analysis}

Hardy-Weinberg equilibrium (HWE), allele and genotype frequency analyses and haplotype analysis were performed using SHEsis (http://shesisplus.bio-X.cn/SHEsis.html). SNPs with a $\mathrm{p}$ value less than 0.001 were considered as a departure from the HWE. ${ }^{28}$ The interaction between the markers was analyzed using multifactor dimensionality reduction (MDR) software (version 3.0.2 (Computational Genetics Laboratory,
University of Pennsylvania, State College, PA, USA). Power analysis was performed using the Quanto program (Version 1.2.3, https://bio.tools/QUANTO). All tests were two-tailed, and the significance level was set at 0.05 .

\section{RESULTS}

The PPI network encoded by CHRNA3, CHRNA4, CHRNA5, CHRNA7 and schizophrenia susceptibility genes consists of 339 nodes and 191 edges. Each node represents a protein, and the edges represent interactions between proteins. As shown in the Figure 1, the proteins of CHRNA3, CHRNA4, CHRNA5, and CHRNA7 interact with the proteins encoded by the four schizophrenic-related susceptibility genes, which are iron-responsive element-binding protein 2 (IREB2), hydroxylysine kinase (HYKK9), proteasome subunit alpha type4 (PSMA4), neuronal acetylcholine receptor subunit beta- 4 (CHRNB4).

For all the SNPs investigated in the present study, there was no deviation from the Hardy-Weinberg equilibrium ( $\mathrm{p}>0.05)$.

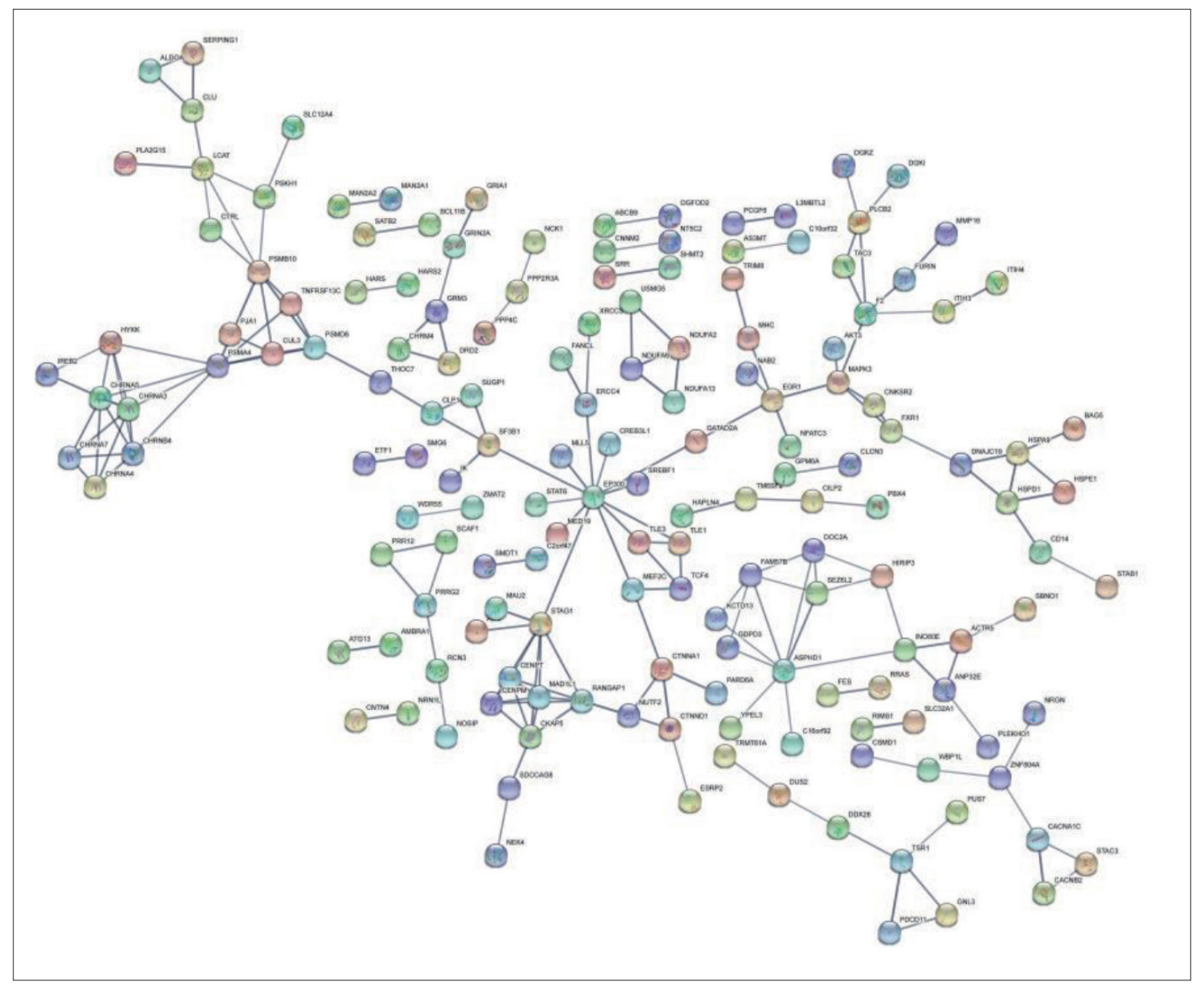

Figure 1. PPI network analysis of $n A C h R$ proteins and 350 proteins encoded by schizophrenia susceptibility genes. The circle represents the protein encoded by the corresponding gene, the pattern within the circle represents the structure of the protein, and the line represents the PPI. PPI, protein-protein interaction. 
There was no significant difference in either allele or genotype frequencies between the patients and controls in single marker analysis (Table 1). The haplotype structures of 4 SNPs (rs1317286, rs16969968, rs6494212, and rs684513) distributed on chromosome 15 were analyzed. The red square of the linkage disequilibrium (LD) block indicated a strong linkage between the marker rs16969968 and rs1317286 $\left(D^{\prime}=0.96\right)$, rs16969968 and rs684513 (D'=0.95), as shown in Figure 2. The haplotypes construct by markers 4 SNPs were not associated with schizophrenia (Table 2).

In interaction analysis, a significant association was revealed between models made up of rs1317286, rs1044396, rs6494212, and rs684513 and schizophrenia $(\mathrm{p}<0.05)$. Testing Balanced accuracy of the model is 0.4864 , the result of Cross-Validation consistency is 10/10 (Table 3). Power analysis showed greater than $80 \%$ power (alpha $=0.05$ ) for schizophrenia samples with an $\mathrm{OR}=1.5$, under the assumption of a log-additive model and disease prevalence of $1 \%$. Thus, the likelihood of type II error appeared below.

\section{DISCUSSION}

This study does not demonstrate a significant genetic difference in 5 markers in $\mathrm{AAChR}$ genes between schizophrenia patients and healthy controls in the single marker analysis. The haplotypes constructed by these four markers of CHRNA3, CHRNA5, and CHRNA7 gene were not associated with schizophrenia either. However, analyzing the interaction of the SNPs by MDR, we found that the interaction between the four-SNP model, rs1317286, rs6494212, rs684513, and rs1044396 produced a significant risk effect for schizophrenia.

We selected four nicotinic receptor subunit genes in this study. CHRNA4 is located on chromosome 20 at position 20q13.2-q13.3. Some SNPs of CHRNA4 were found to be associated with many psychiatric disorders, such as schizophrenia, Alzheimer's disease (AD), attention-deficit/hyperactivity disorder (ADHD), Parkinson's disease (PD), and nicotine dependence. ${ }^{29,30}$ More specifically, a single nucleotide polymorphism in the CHRNA4 gene (rs1044396) appears to affect visual and auditory function, affecting speed and attention. ${ }^{31}$ CHRNA3 is located on chromosome 15 at position 15q24. Recently, rs1051730 and rs1317286 of CHRNA3 were found to be associated with prepulse inhibition, which is the endophenotype of schizophrenia. ${ }^{23}$ CHRNA5 is located on chromosome 15 at position $15 \mathrm{q} 24$. Association has been reported between schizophrenia and SNPs of the CHRNA5. ${ }^{32}$

The CHRNA7 located on chromosome 15q13-q14 was

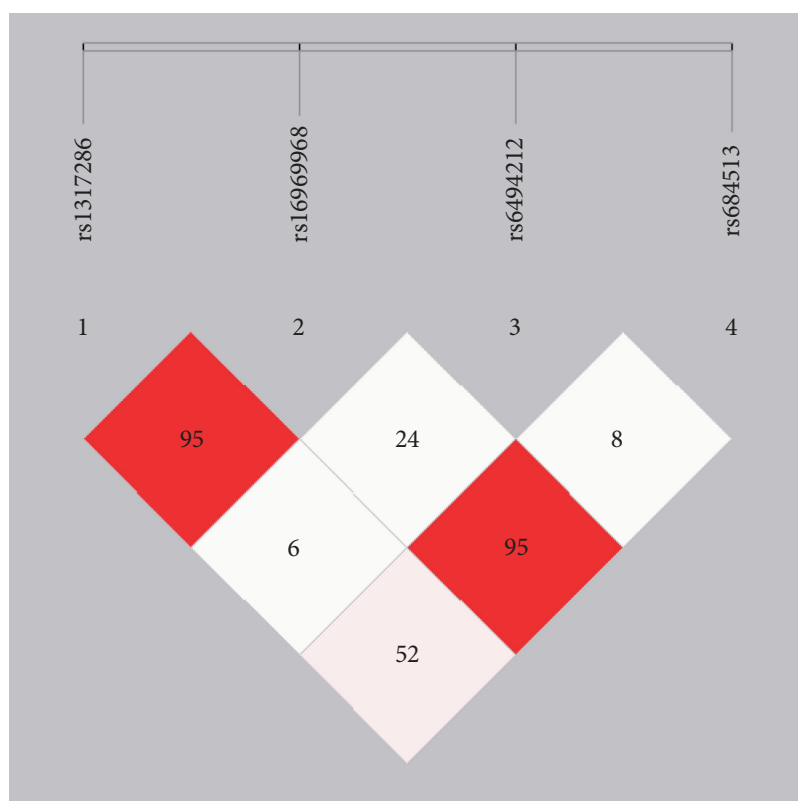

Figure 2. Linkage disequilibrium (LD) pattern of four Single Nucleotide Polymorphisms (SNPs) in schizophrenia patients and controls. Diagram of the haplotypes and pair-wise LD of the 4 SNP loci (rs1317286, rs16969968, rs6494212, and rs684513). The red color indicates a greater LD, and the lighter color indicates a lower LD.

Table 2. Haplotype association with schizophrenia

\begin{tabular}{cccc}
\hline \multirow{2}{*}{ Haplotype } & \multicolumn{2}{c}{ Frequency } & \multirow{2}{*}{ p-value } \\
\cline { 2 - 3 } & Case & Control & \\
\hline A G C C & 0.628 & 0.623 & 0.954689 \\
A G C G & 0.202 & 0.203 & 0.856426 \\
A G T C & 0.055 & 0.058 & 0.708562 \\
G G C C & 0.058 & 0.053 & 0.563916 \\
\hline
\end{tabular}

Table 1. Distributions of alleles and genotypes for the Single Nucleotide Polymorphisms (SNPs) in cases and controls

\begin{tabular}{|c|c|c|c|c|c|c|c|c|c|}
\hline \multirow{2}{*}{ SNPs } & \multirow{2}{*}{ Allele } & \multicolumn{2}{|c|}{ No. of samples } & \multirow{2}{*}{$\mathrm{p}$-value } & \multirow{2}{*}{ Genotype } & \multicolumn{2}{|c|}{ No. of samples } & \multirow{2}{*}{ HWE p-value* } & \multirow{2}{*}{$\mathrm{P}_{\text {adjust-value }}$} \\
\hline & & Case & Control & & & Case & Control & & \\
\hline rs1317286 & $\mathrm{A} / \mathrm{G}$ & $1,876 / 188$ & $1,478 / 150$ & 0.912 & $\mathrm{AA} / \mathrm{AG} / \mathrm{GG}$ & $850 / 176 / 6$ & $673 / 132 / 9$ & 0.381 & 0.420 \\
\hline rs1044396 & $\mathrm{G} / \mathrm{A}$ & $1,539 / 505$ & $1,213 / 407$ & 0.772 & AA/AG/GG & $58 / 389 / 575$ & $51 / 305 / 454$ & 0.981 & 0.854 \\
\hline rs16969968 & $\mathrm{G} / \mathrm{A}$ & $1,997 / 43$ & $1,573 / 43$ & 0.273 & AA/AG/GG & 2/39/979 & $0 / 43 / 765$ & 0.437 & 0.195 \\
\hline rs6494212 & $\mathrm{C} / \mathrm{T}$ & $1,890 / 174$ & $1,473 / 143$ & 0.612 & CC/CT/TT & $865 / 160 / 7$ & $673 / 127 / 8$ & 0.912 & 0.752 \\
\hline rs684513 & $\mathrm{C} / \mathrm{G}$ & $1,578 / 482$ & $1,234 / 388$ & 0.711 & CC/CG/GG & $599 / 380 / 51$ & $471 / 292 / 48$ & 0.759 & 0.641 \\
\hline
\end{tabular}

*Hardy-Weinberg p-value was calculated in the controls group 
Table 3. Multilocus interaction model by MDR method

\begin{tabular}{lcccc}
\hline \multicolumn{1}{c}{ Model } & $\begin{array}{c}\text { Training balanced } \\
\text { accuracy }\end{array}$ & $\begin{array}{c}\text { Testing balanced } \\
\text { accuracy }\end{array}$ & $\begin{array}{c}\text { p-value } \\
\text { Cross-validation } \\
\text { consistency }\end{array}$ \\
\hline rs684513 & 0.5098 & 0.4620 & 0.3922 & $4 / 10$ \\
rs1044396, rs684513 & 0.5203 & 0.4609 & 0.0944 & $4 / 10$ \\
rs1044396, rs16969968, rs684513 & 0.5317 & 0.4807 & 0.0065 & $6 / 10$ \\
rs1317286, rs1044396, rs16969968, rs684513 & 0.5476 & 0.4864 & $<0.0001$ & $10 / 10$ \\
\hline
\end{tabular}

MDR, multifactor dimensionality reduction

first genetically linked to the P50 auditory sensory processing deficit in schizophrenia. ${ }^{15}$ Associations also have been reported between nicotine dependence and SNPs of the CHRNA7 (rs1909884, rs883473, rs2337980, rs6494212, rs904951, rs4779565, and rs10438287), and rs6494212 of CHRNA7 was reported to be associated with schizophrenia recently. ${ }^{33}$

The PPI network found that the proteins of CHRNA3, CHRNA4, CHRNA5, and CHRNA7 interact with the proteins encoded by four schizophrenic-related susceptibility genes (REB2, HYKK9, PSMA4, and CHRNB4). To some extent, it suggested that $\mathrm{nAChR}$ protein might be involved in or affect the molecular pathway of schizophrenic-related susceptibility genes. The above findings suggested that the nAChR genes are possibly involved in the pathogenesis of schizophrenia. Therefore, we set out to study the association between schizophrenia and nAChR genes. But the present study revealed no association between schizophrenia and SNPs in the single-marker analysis.

Schizophrenia is a complex psychiatric disease is associated with numerous genes that individually confer small and incremental risk to the phenotype, and epistatic interactions can enhance the pathogenic effects of alleles. ${ }^{34,35}$ In our study, the results of MDR revealed that polymorphisms in CHRNA3 (rs1317286), CHRNA7 (rs6494212), CHRNA5 (rs684513), and CHRNA4 (rs1044396) have significant gene-gene interactions in schizophrenia. These SNPs have been previously suggested to contribute to schizophrenia. susceptibility. These results demonstrate an accumulation of evidence for the role of an interaction between CHRNA3, CHRNA4, CHRNA5 and CHRNA7 may conferring susceptibility to schizophrenia.

There are several possible explanations for the lack of association between schizophrenia and the selected SNPs, noted as follows: Firstly, SNPs of the nAChR genes we selected are unlikely to play a substantial role in conferring susceptibility to schizophrenia in the Han Chinese population. But, associations of SNPs with schizophrenia or endophenotypes of schizophrenia have been reported across different ethnic groups. This could be explained by population differences where heterogeneous effects of the same polymorphism are caused by differences in genetic background. Secondly, schizophrenia is a complex disorder, in which multiple loci act jointly to confer susceptibility for disease. It is believed that schizophrenia involves multiple genetic and environmental causes. The genetic variation subjected to scrutiny in this study may provide only a limited contribution to schizophrenia. There is still a possibility that association occurs in subsets of schizophrenic patients. Further studies in a largely homogenous population are needed. Interestingly, when the SNPs were analyzed together, the interaction between four SNPs produced a significant risk effect for schizophrenia. Further research including family-based studies is needed to investigate the role of these genes in schizophrenia and the functions of the brain.

One limitation of this study is that we explored only one variant for CHRNA3, CHRNA4, and CHRNA7 and two variants for CHRNA5. Thus additional polymorphisms in these four subunit genes should be genotyped to confirm the interaction. Another limitation is that we did not analyze gene-environmental interaction in this study.

In conclusions, the results of the present study do not support polymorphisms selected as a major genetic susceptibility factor in schizophrenia in the single marker analysis. However, found that a four-SNP model had an important effect on schizophrenia by MDR analysis. Our findings may represent a significant genetic clue for the etiology of schizophrenia and thus further studies are required to uncover the exact role of genes in schizophrenia.

\section{Availability of Data and Material}

The datasets generated or analyzed during the study are available from the corresponding author on reasonable request.

\section{Conflicts of Interest}

The authors have no potential conflicts of interest to disclose.

\section{Author Contributions}

Conceptualization: Yuan-yuan Li. Data curation: Hua-fang Li. Formal analysis: Yuan-yuan Li. Funding: Hua-fang Li, Shun-Ting Yu. Investigation: Yuan-yuan Li. Methodology: Shun-ying Yu. Project administration: Hua-fang Li. Resources: Guan-jun Li. Software: Zhou-ye Wang. Supervision: Zhou-ye Wang, Hua-fang Li. Validation: Hua-fang Li. Visualization: Hua-fang Li. Writing_original draft: Yuan-yuan Li, Rui-jie Geng. Writing—review \&editing: Yuan-yuan Li, Rui-jie Geng. 


\section{ORCID iDs}

Yuan-yuan Li

Rui-jie Geng

Shun-ying Yu

Guan-jun Li

Zhou-ye Wang

Hua-fang Li

https://orcid.org/0000-0002-2956-4163 https://orcid.org/0000-0001-9152-9425 https://orcid.org/0000-0001-7929-204X https://orcid.org/0000-0002-9900-8963 https://orcid.org/0000-0002-5565-9780 https://orcid.org/0000-0002-4357-7614

\section{Funding Statement}

The authors are deeply grateful to all participants. This work was supported by the Shanghai clinical research center for mental health (No. 19MC1911100) and the GCP for psychotropics of Collaborative Innovation Center for Translational Medicine at Shanghai Jiao Tong University School of Medicine (No.TM202016).

\section{REFERENCES}

1. Richetto J, Meyer U. Epigenetic modifications in schizophrenia and related disorders: molecular scars of environmental exposures and source of phenotypic variability. Biol Psychiatry 2021;89:215-226.

2. Hasan A, Falkai P, Lehmann I, Gaebel W. Schizophrenia. Dtsch Arztebl Int 2020;117:412-419.

3. Dervaux A, Laqueille X. [Smoking and schizophrenia: epidemiological and clinical features]. Encephale 2008;34:299-305.

4. Šagud M, Vuksan-Ćusa B, Jakšić N, Mihaljević-Peleš A, Rojnić Kuzman M, Pivac N. Smoking in schizophrenia: an updated review. Psychiatr Danub 2018;30:216-223.

5. Kozak K, George TP. Pharmacotherapy for smoking cessation in schizophrenia: a systematic review. Expert Opin Pharmacother 2020;21:581590.

6. Avissar M, Xie S, Vail B, Lopez-Calderon J, Wang Y, Javitt DC. Metaanalysis of mismatch negativity to simple versus complex deviants in schizophrenia. Schizophr Res 2018;191:25-34.

7. Wolf A, Ueda K, Hirano Y. Recent updates of eye movement abnormalities in patients with schizophrenia: a scoping review. Psychiatry Clin Neurosci 2021;75:82-100.

8. Wong DF, Kuwabara H, Horti AG, Roberts JM, Nandi A, Cascella N, et al. Brain PET imaging of $\alpha 7$-nAChR with [18F]ASEM: reproducibility, occupancy, receptor density, and changes in schizophrenia. Int J Neuropsychopharmacol 2018;21:656-667.

9. D'Souza MS, Markou A. Schizophrenia and tobacco smoking comorbidity: $\mathrm{nAChR}$ agonists in the treatment of schizophrenia-associated cognitive deficits. Neuropharmacology 2012;62:1564-1573.

10. Parikh V, Kutlu MG, Gould TJ. nAChR dysfunction as a common substrate for schizophrenia and comorbid nicotine addiction: current trends and perspectives. Schizophr Res 2016;171:1-15.

11. Wang S, Che T, Levit A, Shoichet BK, Wacker D, Roth BL. Structure of the $\mathrm{D} 2$ dopamine receptor bound to the atypical antipsychotic drug risperidone. Nature 2018;555:269-273.

12. Morel C, Fernandez SP, Pantouli F, Meye FJ, Marti F, Tolu S, et al. Nicotinic receptors mediate stress-nicotine detrimental interplay via dopamine cells' activity. Mol Psychiatry 2018;23:1597-1605.

13. Faure P, Tolu S, Valverde S, Naudé J. Role of nicotinic acetylcholine receptors in regulating dopamine neuron activity. Neuroscience 2014;282: 86-100.

14. Sinkus ML, Graw S, Freedman R, Ross RG, Lester HA, Leonard S. The human CHRNA7 and CHRFAM7A genes: a review of the genetics, regulation, and function. Neuropharmacology 2015;96:274-288.

15. Bertelsen B, Oranje B, Melchior L, Fagerlund B, Werge TM, Mikkelsen JD, et al. Association study of CHRNA7 promoter variants with sensory and sensorimotor gating in schizophrenia patients and healthy controls: a danish case-control study. Neuromolecular Med 2015;17:423-430.

16. Kunii Y, Zhang W, Xu Q, Hyde TM, McFadden W, Shin JH, et al. CHRNA7 and CHRFAM7A mRNAs: co-localized and their expression levels altered in the postmortem dorsolateral prefrontal cortex in major psychiatric disorders. Am J Psychiatry 2015;172:1122-1130.

17. Kalmady SV, Agrawal R, Venugopal D, Shivakumar V, Amaresha AC, Agarwal SM, et al. CHRFAM7A gene expression in schizophrenia: clinical correlates and the effect of antipsychotic treatment. J Neural Transm (Vienna) 2018;125:741-748.

18. Freedman R. $\alpha 7$-nicotinic acetylcholine receptor agonists for cognitive enhancement in schizophrenia. Annu Rev Med 2014;65:245-261.

19. Shi J, Wang Z, Tan Y, Fan H, An H, Zuo L, et al. CHRNA4 was associated with prepulse inhibition of schizophrenia in Chinese: a pilot study. Cogn Neuropsychiatry 2016;21:156-167.

20. Hyde M, Choueiry J, Smith D, de la Salle S, Nelson R, Impey D, et al. Cholinergic modulation of auditory $\mathrm{P} 3$ event-related potentials as indexed by CHRNA4 and CHRNA7 genotype variation in healthy volunteers. Neurosci Lett 2016;623:36-41.

21. Lee SH, Ahn WY, Seweryn M, Sadee W. Combined genetic influence of the nicotinic receptor gene cluster CHRNA5/A3/B4 on nicotine dependence. BMC Genomics 2018;19:826.

22. Petrovsky N, Ettinger U, Kessler H, Mössner R, Wolfsgruber S, Dahmen $\mathrm{N}$, et al. The effect of nicotine on sensorimotor gating is modulated by a CHRNA3 polymorphism. Psychopharmacology 2013;229:31-40.

23. Petrovsky N, Quednow BB, Ettinger U, Schmechtig A, Mössner R, Collier DA, et al. Sensorimotor gating is associated with CHRNA3 polymorphisms in schizophrenia and healthy volunteers. Neuropsychopharmacology 2010;35:1429-1439.

24. Hong LE, Yang X, Wonodi I, Hodgkinson CA, Goldman D, Stine OC, et al. A CHRNA5 allele related to nicotine addiction and schizophrenia. Genes Brain Behav 2011;10:530-535.

25. Oh S, Lee J, Kwon MS, Weir B, Ha K, Park T. A novel method to identify high order gene-gene interactions in genome-wide association studies: gene-based MDR. BMC Bioinformatics 2012;13(Suppl 9):S5.

26. Biological insights from 108 schizophrenia-associated genetic loci. Nature 2014;511:421-427.

27. Pardiñas AF, Holmans P, Pocklington AJ, Escott-Price V, Ripke S, Carrera N, et al. Common schizophrenia alleles are enriched in mutationintolerant genes and in regions under strong background selection. Nature Genet 2018;50:381-389.

28. Wang HZ, Bi R, Zhang DF, Li GD, Ma XH, Fang Y, et al. Neprilysin confers genetic susceptibility to Alzheimer's disease in Han Chinese. Mol Neurobiol 2016;53:4883-4892.

29. Eggert M, Winterer G, Wanischeck M, Hoda JC, Bertrand D, Steinlein $\mathrm{O}$. The nicotinic acetylcholine receptor alpha 4 subunit contains a functionally relevant SNP Haplotype. BMC Genet 2015;16:46.

30. Becchetti A, Grandi LC, Colombo G, Meneghini S, Amadeo A. Nicotinic receptors in sleep-related hypermotor epilepsy: pathophysiology and pharmacology. Brain Sci 2020;10:907.

31. Mobascher A, Diaz-Lacava A, Wagner M, Gallinat J, Wienker TF, Drichel $\mathrm{D}$, et al. Association of common polymorphisms in the nicotinic acetylcholine receptor alpha4 subunit gene with an electrophysiological endophenotype in a large population-based sample. PLoS One 2016;11: e0152984.

32. Zhan D, Yao Q, Fu S, Liu X, Zhou J, Chen D, et al. Impact of CHRNA5 polymorphisms on the risk of schizophrenia in the Chinese Han population. Mol Genet Genomic Med 2019;7:e869.

33. Saccone NL, Schwantes-An TH, Wang JC, Grucza RA, Breslau N, Hatsukami D, et al. Multiple cholinergic nicotinic receptor genes affect nicotine dependence risk in African and European Americans. Genes Brain Behav 2010;9:741-750.

34. Su Y, Yang L, Li Z, Wang W, Xing M, Fang Y, et al. The interaction of ASAH1 and NGF gene involving in neurotrophin signaling pathway contributes to schizophrenia susceptibility and psychopathology. Prog Neuropsychopharmacol Biol Psychiatry 2021;104:110015.

35. Hu G, Yang C, Zhao L, Fan Y, Lv Q, Zhao J, et al. The interaction of NOS1AP, DISC1, DAOA, and GSK3B confers susceptibility of early-onset schizophrenia in Chinese Han population. Prog Neuropsychopharmacol Biol Psychiatry 2018;81:187-193. 